\title{
Native Apps versus Web Apps: Which Is Best for Healthcare Applications?
}

\author{
Kirusnapillai Selvarajah ${ }^{1}$, Michael P. Craven ${ }^{1}$, Adam Massey ${ }^{2}$, John Crowe ${ }^{1}$, \\ Kavita Vedhara ${ }^{2}$, and Nicholas Raine-Fenning ${ }^{3}$ \\ ${ }^{1}$ The University of Nottingham, Electrical Systems \& Optics Research Group, \\ Faculty of Engineering, University Park, Nottingham NG7 2RD, United Kingdom \\ \{Kirusnapillai.Selvarajah, Michael.Craven, \\ John. Crowe \} @nottingham.ac.uk \\ ${ }^{2}$ The University of Nottingham, School of Community Health Sciences, \\ University Park, Nottingham NG7 2RD, United Kingdom \\ \{mjxajm, kavita.vedhara\} @nottingham.ac.uk \\ ${ }^{3}$ The University of Nottingham, Division of Obstetrics \& Gynaecology, \\ School of Clinical Sciences, Queen's Medical Centre (QMC), \\ Nottingham, NG7 2UH, United Kingdom \\ nick.raine-fenning@nottingham.ac.uk
}

\begin{abstract}
Smartphone applications (Apps) provide a new way to deliver healthcare, illustrated by the fact that healthcare Apps are estimated to make up over $30 \%$ of new Apps currently being developed; with this number seemingly set to increase as the benefits become more apparent. In this paper, using the development of an In Vitro Fertilisation (IVF) treatment stress study App as the exemplar, the alternatives of Native App and Web App design and implementation are considered across several factors that include: user interface, ease of development, capabilities, performance, cost, and potential problems. Development for iOS and Android platforms and a Web App using JavaScript and HTML5 are discussed.
\end{abstract}

Keywords: Web Apps, Native Apps, mHealth, Ecological Momentary Assessment, User Interface, User Experience, JavaScript, HTML5, Android, iOS.

\section{Introduction}

Smartphones are becoming the central computer and communication device in people's lives offering multiple means of interaction via many built-in sensor types such as accelerometers, gyroscopes, GPS, cameras and microphone [1]. Most Smartphones are now integrated with Wi-Fi, Wi-Fi Direct, Bluetooth and NFC networking capabilities which also enable them to connect to external sensors, devices and various networks. These capabilities are enabling new applications in, for example, the area of healthcare $[2,3]$, transportation [4], retail, banking and environmental monitoring. Healthcare Apps build upon earlier work in telehealth, mobile computing and pervasive computing in healthcare settings [5, 6]. Application areas for Smartphones in healthcare include education and 
awareness, remote data collection, communication and training for healthcare workers, disease and epidemic outbreak tracking and diagnostic and treatment support [7].

This paper describes the design and development of a Web App and two Native Apps for use in a prospective observational study whose aim is to examine what psychosocial factors influence distress levels in patients throughout the duration of one cycle of In Vitro Fertilisation (IVF) treatment. The study uses a number of questionnaires which ask patients to report their health status in relation to their infertility and its treatment. The patients need to be signalled to complete the questionnaire at different time points throughout the treatment process. To support this, a Smartphone application (IVF App) has been designed so that, at each selected time point, the patient receives a reminder to complete the set of questionnaires. One of the questionnaires has different questions presented on different days. Each time they complete the questionnaires, response data is sent to a server for analysis.

\section{IVF Treatment Stress Study}

This study aims to examine what psychosocial factors may predict levels of distress in women who are undergoing a cycle of In Vitro Fertilisation. A methodological weakness of past research within this area relates to the timing and implementation of the distress measures which, to date, have tended to be administered at single time points, often before treatment commences, using conventional paper-based methods [8]. A cycle of IVF treatment generally consists of three stages: down regulation; stimulation; and embryo transfer. Each stage is characterised by different physical and psychological demands upon the patient. For example, the down regulation and stimulation stages routinely entail self-administering drugs via injection, which occurs whilst patients ready themselves for the emotional and physical burdens of undergoing the embryo transfer surgical procedure. As such, each stage of the treatment is considered somewhat separate and indeed it does appear that they differ in the extent to which they lead to patients experiencing distress. Therefore, administering psychosocial measures at a single time point fails to adequately capture the considerable heterogeneity in which patients will respond to different aspects of the treatment process.

The over-reliance on single time point measures within the literature has been based on the premise that it is necessary to reduce the degree of potential burden that successive paper-based questionnaires or diaries may confer. Therefore, it is crucial that novel approaches must be user-friendly so that they optimally capture psychological distress throughout the stages of the IVF process in ways that minimise this burden. Alternatives to paper-based diaries include Personal Digital Assistants (PDA) and more recently Smartphones. The methodology underpinning the use of such methods is commonly referred to as Ecological Momentary Assessment (EMA) [9].

Within this study, patients are signalled every two days at varying time points. The facility to vary the time points of patient signalling confers an advantage in that certain moods may become entrained to particular routines and times of day [10] and thus impact upon the levels of distress that are subsequently reported. A further advantage of a flexible signalling facility over and above the use of paper based methods is that the response delay (i.e. the time it takes to respond to the signal and complete the task) may also provide an indirect measure of response reliability. 
A response that is too long after the initial signal is susceptible to retrospective recall bias and thus compromises the central tenet of EMA $[9,11]$. Another significant advantage of the method is that data is transferred to a secure server and thus streamlines the data collection and storage.

\section{IVF App Design}

In order to design the IVF App, it was necessary to consider the different viewpoints of the developers, clinical researchers and users (i.e. patients). First, a small survey was conducted to gather information from the IVF fertility clinic at the Queens Medical Centre in Nottingham. For this a questionnaire was designed to examine smartphone usage in order to gather information for the IVF App design and development. 76 females attending the fertility clinic for IVF treatment completed the questionnaire and the findings are presented in Table 1 below.

Table 1. User phone models survey result

\begin{tabular}{|l|c|c|}
\hline Phone Model & Frequency & Percentage \\
\hline iOS & 31 & 41 \\
\hline Android & 25 & 33 \\
\hline Symbian & 11 & 14 \\
\hline BlackBerry OS & 9 & 12 \\
\hline
\end{tabular}

As reported in Table 1, the majority of the patients use the iOS and Android smartphone platforms, representing $74 \%$ of the total. It was therefore decided that the IVF App should be developed on both platforms to optimise the number of patients included in the study. Furthermore $80 \%$ of patients reported familiarity with Smartphone Apps and use of the phone for internet access. This is important because internet access is required for transfer of the patient responses to the secure server. A related question therefore was whether internet coverage was included in the patient's contract to determine if this would result in additional expense to them. $82 \%$ of patients reported that their internet coverage was covered by their contract agreement with their air time provider. To inform the use of any signalling mechanism patients were asked whether or not they used an alarm facility on their mobile phones with $92 \%$ reporting that they did, so indicating that it could be incorporated into the IVF app design.

The purpose of the IVF App is to measure distress by asking the patients about their current mood and feelings of stress with measures taken every two days. The patients are asked first "How stressed are you feeling right now" and then to indicate whether this stress is related to their fertility issue in general and/or the IVF treatment itself. These questions are designed to measure conscious mood, that is, feelings relating to fertility and the treatment that the patient is aware of. The second task delivered 
via the IVF App is designed to capture the extent of unconscious stress the patient may be experiencing [8].

One instrument designed to capture unconscious mood is the IPANAT [13] that requires respondents to rate the extent to which a nonsense word represents or sounds like a particular descriptive. The descriptives used within this IVF study were adapted from those used in the original IPANAT so as to represent feelings more closely related to the experience of infertility. Patients are asked to what extent the nonsense words SAFME, VIKES, TUNBA, TALEP, BELINI and SUKOV 'sound like' the descriptives CALM, TENSE, UPSET, RELAXED, CONTENT and WORRIED. Only one nonsense word from the list of six (SAFME, VIKES, TUNBA, TALEP, BELINI and SUKOV) was presented to the patients every two days. This was to avoid presenting the full IPANAT which it was considered would have been a burden to the patient. Presenting one word at each time point also allows for changes in mood to be assessed over time.

\subsection{IVF Web App Implementation}

Web Apps are application programs that can be accessed over the internet through a Smartphone's web browser. Web Apps enable information processing functions to be initiated remotely on the server. A Typical Web App consists of: a client layer; application layer (on a server); and database layer. Based on the survey, patients used different smartphone platforms such as iOS, Android, Symbian and BlackBerry OS. Therefore we initially decided to investigate the possibility of building a Web App to support all phones with a web browser which would also reduce development time.

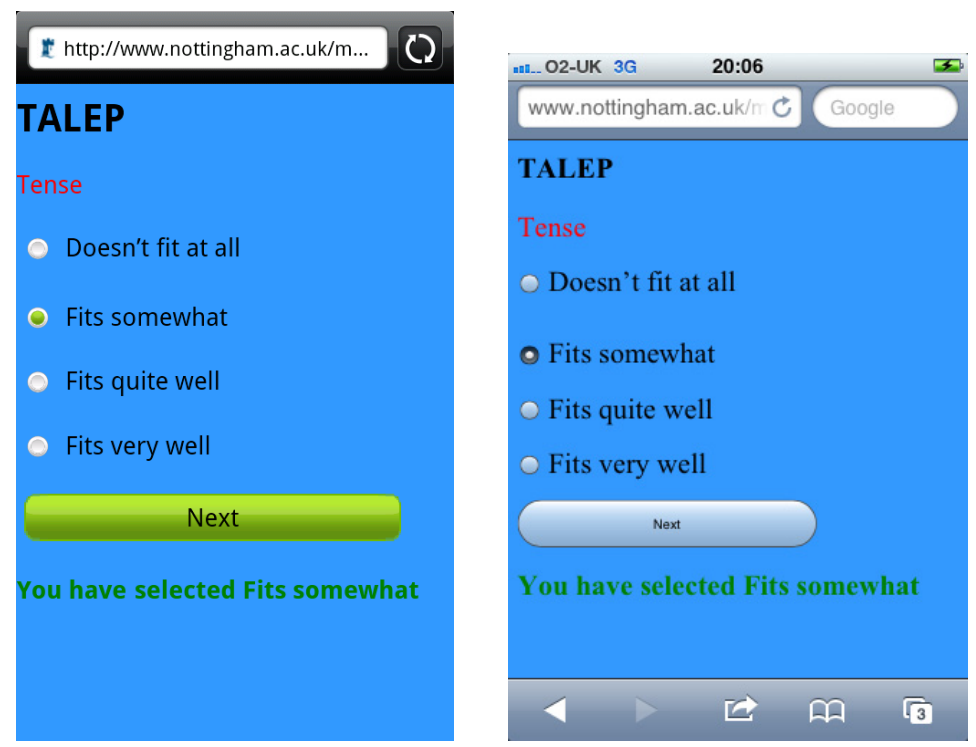

Fig. 1. Screenshots of IVF Web App implementation on Android and iPhone 
Screenshots of the Web App implementation (with Android and iPhone displays) are shown in Fig 1. The basic questionnaire of the IVF treatment stress study was implemented using HTML5, JavaScript and jQuery Mobile but it was not possible to implement a sensible continuous signalling mechanism for different time points, because Smartphone notifications and persistent data storage features are not normally accessible through web technologies (i.e. HTML5, JavaScript). Another possible way of providing signalling is via SMS from a server although control over the response timing is lost. Furthermore, a Web App cannot work offline and mobile internet connections (GPRS, GSM, EDGE, HSPA etc.) are often unreliable inside buildings or in the urban canyon environment and Wi-Fi is not always available or accessible. One of the main requirements for the IVF App is that it should provide signals (alerts) to the patient in a predefined time irrespective of the mobile network coverage. Furthermore, there was a concern from the NHS ethics committee about the usage of SMS text messaging due to data confidentiality. However, since some kind of signalling is still required to prompt the completion of the questionnaires it was considered better to use to an integrated signalling mechanism inside the Native App rather than a separate signalling mechanism with a Web App. Consequently the Web App implementation of the IVF study was abandoned.

\subsection{IVF Native App Implementation}

Native Apps only work on the proprietary smartphone operating system for which they are developed (e.g. iOS, Android, Windows Mobile) and each smartphone platform has its own development process (e.g., Xcode, Eclipse, Windows Mobile Development Tool) and uses a different programming language (e.g. Objective $\mathrm{C}$ for iPhone, Java for Android Phones and C\# for Windows Mobile). Native Apps can be stand-alone applications without any need server interaction. The following sections briefly discuss IVF Native App implementations for Android and iOS.
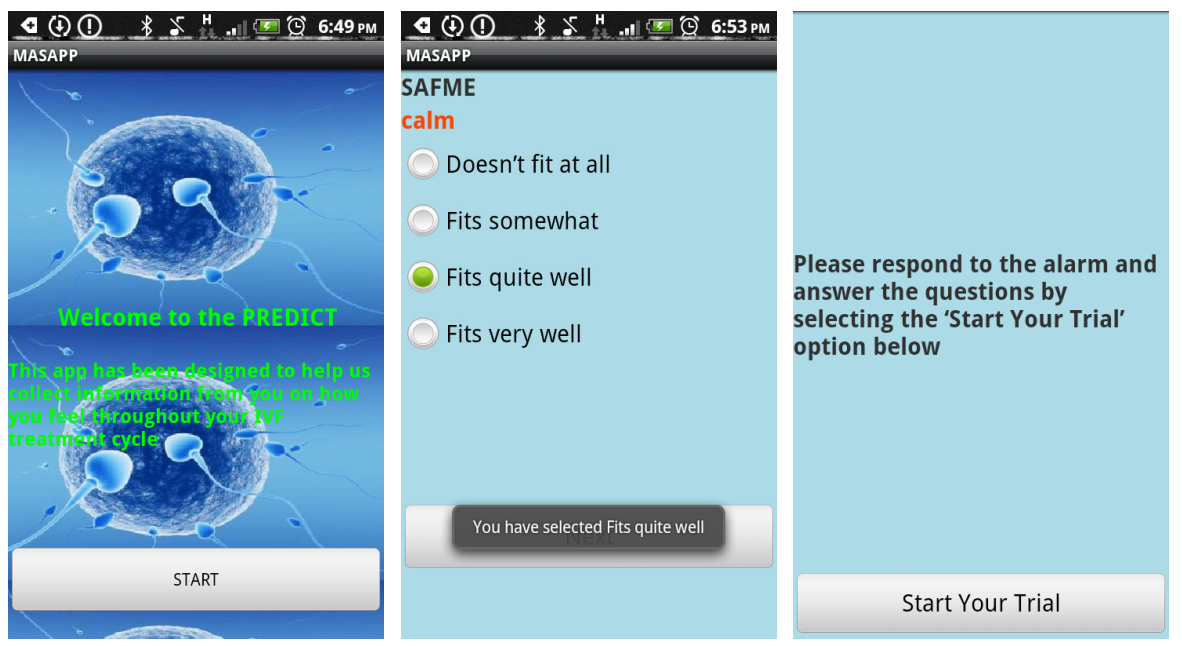

Fig. 2. Screenshot of the Android Native IVF App 


\section{Android Native App Implementation}

The Android IVF Native App was developed using the Eclipse Android Development Tool (ADT) plugin with Java programming language. The common user interface elements (Buttons, TextView, Radio Buttons, Message Notification (Toast) and Activity) were used from the Android Library [14]. Android AlarmManager functionalities were used to provide signalling at specified time points. At each time point the user responses (with timestamps) are transferred to the secure server when a connection becomes available, or if there is no internet connection, the data can be recorded in the phone's memory.

\section{iOS Native App Implementation}

The iPhone IVF native App was developed using Xcode with the Objective-C programming language with user interface elements (Buttons, Labels, TextFields, Local Notification and View Controller) from the iOS Cocoa Touch library [15]. As no Radio Button objects are provided with Cocoa Touch, standard Button Objects were customised to produce a 'Radio button' object. The Local notification mechanism provided by the iOS (UILocalNotification) was used to provide a continuous signalling mechanism for different time points. Similar to the Android IVF Native App, at each time point the user responses (with timestamps) are transferred to the secure server and are also recorded in the phone's memory. Screenshots are presented in Fig. 3 from the first version of the iOS IVF Native App implementation on an iPhone (hence further tests may result in a slightly different output).
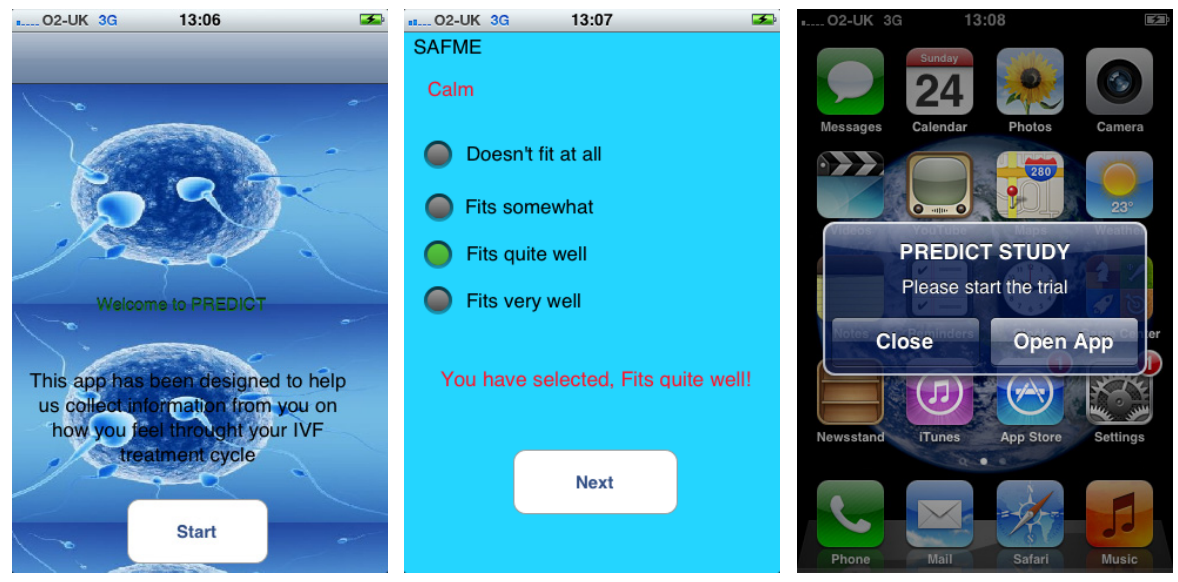

Fig. 3. Screenshots of the iPhone IVF Native App

\section{Discussion and Conclusions}

Native Apps are able to leverage elements of their native operating system so that they have a more responsive and attractive User Interface and can be run offline. If an internet connection is necessary then they can resume the necessary action with the server (e.g. file uploading) when the connection is successfully restored. The rest of the time, the App can run stand-alone. 
Native Apps can more effectively use phone hardware such as accelerometers, gyroscopes, GPS, cameras and microphones as well as phone software applications such as contacts and calendar. The IVF Native App implementations used AlarmManager functionalities, Local Notification features and File Storage access which are not normally possible in the case of a Web App implementation using JavaScript and HTML5. Also, importantly, some healthcare Apps need a high rate of sampling of the sensor reading which can be achieved using Native processing (e.g. Java Native Interface in Android) whilst others may need to interface with external sensors with wireless communication technologies where access to the native networking related native APIs (Application Programming Interface) is necessary.

However, since they only work on the platform they were designed for, porting a Native App across different platforms takes up more resources for code development and testing time. In some instances there may need to be a complete re-redesign of the App since different host systems provide different restrictions to the access of hardware and software functions.

Web Apps can run on any mobile web browser and can be developed using JavaScript, HTML5 and CSS3 so that device-specific customisation can often be achieved easily. Because of this, the cost of developing a Web App for use across different platforms is significantly lower than developing multiple Native Apps. This is particularly relevant as hardware fragmentation increases and more platforms must be supported. However, Web Apps cannot have direct access to the hardware features and functionalities of the native operating system of the host platform. They can achieve access to some functions via abstraction using JavaScript and HTML, but generally not all, and this access is platform-dependent. Web Apps are generally less secure than Native Apps as they require constant connection with the server where private information could potentially be compromised.

In the IVF App development, it was decided that a Web App would be less able to deliver a complete and reliable solution for a stress study using EMA compared to that achievable via a Native App.

However, the decision to build either Native Apps or a Web Apps depends not only on technical requirements and functional requirements but budget, resource constraints and time. In some cases, a Web App will be technically possible and so provide the most cost-effective solution. In other cases it will be necessary to expend the extra cost and effort of producing Native Apps.

It should be noted that Hybrid Apps are beginning to emerge that provide cross platform solutions with abstraction via use of the same Native APIs so that developers can write Apps only using JavaScript and HTML5. However, hybrid Apps may not currently provide adequate performance compared to Native Apps due to the overhead introduced by abstraction and HTML rendering in addition to the time to execute the native code, although this may be overcome in the future.

Acknowledgements. MC, KS and JC acknowledge support of this work through the MATCH Programme (EPSRC Grant EP/F063822/1) although the views expressed are entirely their own. The IVF project and involvement of AM, KV and NRF was supported by Nurture Fertility. 


\section{References}

1. Lane, N.D., Miluzzo, E., Lu, H., Peebles, D., Choudhury, T., Campbell, A.T.: A Survey of Mobile Phone Sensing. IEEE Communication Magazine 48(9), 140-150 (2010)

2. Boulos, M.N.K., Wheeler, S., Tavares, C., Jones, R.: How the smartphones are changing the face of the mobile and participatory healthcare: an overview with example from eCAALYX. BioMedical Engineering Online 10(24) (2011)

3. Emerging mHealth: Paths for growth (2013), http://www.pwc.com/en_GX/gx/healthcare/mhealth/assets / pwc-emerging-mhealth-full .pdf (last accessed on February 28, 2013)

4. UC Berkeley/Nokia/NAVTEQ, Mobile Millennium (2011), http: / / traffic.berkeley. edu/ (last accessed on February 28, 2013)

5. Kumar, S., Nilsen, W., Pavel, M., Srivastava, M.: Mobile Health: Revolutionizing Healthcare Through Transdisciplinary Research. IEEE Computer Magazine 46(1), 25-38 (2013)

6. Bardram, J.E., Mihailidis, A., Wan, D. (eds.): Pervasive Computing in Healthcare. CRC Press, Taylor \& Francis Group (November 2006)

7. World Health Organization: mHealth: New horizons for health through mobile technologies: second global survey on eHealth (2011),

http: //www.who.int/publications/goe_mhealth_web.pdf (last accessed February 27, 2013)

8. Matthiesen, S.M.S., Frederiksen, Y., Ingerslev, H.J., Zachariae, R.: Stress, distress and outcome of assisted reproductive technology (ART); A meta analysis. Human Reproduction 26, 2763-2776 (2011)

9. Smyth, J.A., Stone, A.: Ecological momentary assesement in behavioural medicine. Journal of Happiness Studies 4, 35-52 (2002)

10. Smyth, J., Ockenfels, M., Porter, L., Kirschbaum, C., Hellhammer, D., Stone, A.: The association between daily stressors, mood and salivary cortisol secretion. Psychoneuroendocrinology 23, 353-370 (1998)

11. Schwartz, N., Sudman, S.: Autobiographical Memory and the Validity of Retrospective Reports. Springer, New York (1994)

12. Brosschot, J.F., Pieper, S., Thayer, J.F.: Expanding stress theory: Prolonged activiation and perseverative cogntiion. Psychoneuroendocrinology 30, 1043-1049 (2005)

13. Quirin, M., Kazan, M., Kuhl, S.: When nonsense sounds happy or helpless: The implicit positive and negative affect test (IPANAT). Journal of Personality and Social Psychology 3, 500-516 (2009)

14. Android Developer Website, http://developer.android.com/index.html (last accessed February 28, 2013)

15. Apple Developer Website, https: / / developer .apple.com/devcenter/ios/index.action (last accessed February 28, 2013) 